\title{
Analysis of Composition Elements in Oil Painting Sketching
}

\author{
Jialing Shao ${ }^{1}$ Siying Qin ${ }^{1, *}$
}

\author{
${ }^{1}$ Heihe University, Heihe, Heilongjiang 164300, China \\ *Corresponding author. Email: 292995248@qq.com
}

\begin{abstract}
This article focuses on the main design ideas related to the composition of the picture in the process of oil painting sketching, the composition techniques used, and the main points and precautions for sketching composition. Through the specific analysis of the composition of oil painting sketching, it has condensed a composition method that is worth learning from, and provides valuable experience for workers engaged in oil painting art.
\end{abstract}

Keywords: oil painting sketching, composition, elements

\section{INTRODUCTION}

Sketching is an indispensable training content in the process of oil painting modeling. Excellent sketching works are vivid and fresh, and full of strong artistic appeal. Moreover, only through sketching training can the trainees have good oil painting modeling ability and lay a solid foundation for subsequent artistic creation. In the process of oil painting sketching, composition is the most important link, and the quality of the composition directly affects the entire painting process. Without a good picture composition, the work will lose its beauty and soul. Therefore, it is a must to attach great importance to the composition of oil paintings in the process of sketching.

Composition refers to the organization and arrangement of the various components of a painting. This is the condensation of a painter's thoughts and is directly related to personal excitement, painting experience, artistic accomplishment, and personality. In all the steps of sketching, composition is the most easily overlooked component, because the painter always likes to work hard to shape, and despise the composition in sketching, thinking that only when he is creating, he needs to work hard to organize the picture. This is a serious painting misunderstanding. The compositional thought determines the structural form, primary and secondary black and white layout, color block tone processing, tempo and rhythm processing, the use of techniques, the artistic processing of the picture, and other subsequent content in sketching. If someone can master the composition of a painting well, the composition will basically lay the foundation for the success of oil painting sketching. The following is a detailed look at the elements of oil painting sketching composition.

\section{MEANing OF COMPOSITION}

Composition is to purposefully connect what a person see and feel with the knowledge and skills he/her has learned to form a structural system of individual painting language expression. "Construction" refers to the act of actively and intentionally arranging ideas and painting elements together in an orderly manner. "Drawing" refers to the "individual unit" in painting, such as shapes, colors, materials, techniques, etc. "Composition" is the combination of many painting elements, such as points, lines, surfaces, etc., to form a specific, stable structure in which various power elements are cooperating and opposing, changing and harmonious. The composition is inseparable from the "individual unit" in the organization, and it is also inseparable from the action behavior of "construction". The composition of oil painting sketching is the work of organizing individual painting units such as colors, shapes, lines, materials, techniques, etc. It is also an important part of organizing these visual languages into painting languages. Composition is the concrete expression of creative ideas on the screen. It is the first step in oil painting sketching and the most important step in sketching.

\section{ELEMENTS OF COMPOSITION}

Before composing the picture, students should know that: "Abandoning is gaining." They can understand this truth from all the following composition principles of the reason of this saying. The composition in oil painting sketching is diverse, because every sketcher has his own thought and aesthetic orientation, but usually the following composition method is used to organize the picture. The first is to make a small draft composition. In the initial composition, it is a must to use a small draft to complete the composition plan, so it 
is necessary to prepare sketch paper and small color draft materials. After the framing is complete, the painters can draw a small sketch first, and then a small color sketch. They can think of a few more pictures, draw them, compare them, revise them repeatedly, and finalize them. After designing the direction and general steps of the sketching, they can pack it lightly when the final draft is colored, and paint in a very freehand and improvised way, because they already have had a good idea. The sketching drawn in this way will not only have various elements of painting, but also have a strong sense of the scene, and there will be many unexpected highlights, such as lines, brushstrokes, colors, etc., which are the results that can only be achieved after the practice of small draft composition. In short, the painters can sketch the short draft first relaxingly and boldly, and focus the idea on the small draft, so as to lay a solid foundation for future sketching operations on the ground.

\section{A. Observing and framing}

First, the painters can observe and choose an ideal sketching angle. This angle of view must have a certain "artistic inspiration". When taking an angle, it is better to take the points of interest that the painters can feel, such as the expression, taste, light and the surrounding background colors of the sketch object, as the content of their sketching. Because this special feeling touches them, this is often what they are keen to express.

Once they have feelings, they must perform observation and framing analysis. In this step, they must imagine in advance, perceiving first, and have the "foresight" of painting. First of all, when framing a picture, the painters need to respect the first impression they see. The first impression often contains the place where the painters were first moved. This is often the key to whether a work can directly impress and capture the audience's hearts. Therefore, it is necessary to make the framing around the first impression of the so-called first feeling, which is particularly important for the painters.

\section{B. Designing the frame structure}

A frame structure must first be designed in the composition of a sketching work. This structure is not determined by a single painting element, nor does it mean that it can be determined by drawing a subject, such as a sketch object. This is the pre-arrangement of the main structure frame of the picture from the inside to the outside, that is, the composition of the picture. As the dominant motive of composition, the frame structure must form an obvious advantage in the picture, or be the largest in volume, or be repeated many times, including adopting symmetrical or gradual forms to make the theme be highlighted and expressed clearly. The picture is composed of the individual units of each painting. At the same time, these painting elements also form various forms of invisible structural support inside the picture. There are two kinds of them.

1) Dynamic structure: This structure presents a tension that expands outwards, like radiating sunlight, with power overflowing, giving people a sense of openness and movement, showing a trend of centrifugation, or a trend of electric circuit or even multi-channel movement, which taboos being peaceful.

2) Static structure: This structure shows a tendency to converge, the power is cohesive, and the stars converge toward the center like a moon, giving people a sense of calmness and picture. The screen forms the center from the outside to the inside, and establishes the echoes and connections between the various bodies, pursuing peace. In short, the basic structural direction lines or sense of flow connect the main painting elements to the picture.

\section{The layout of the host-guest relationship}

When composing the layout, the relationship between the host and the guest should be straightened. The layout is especially important when the painters combine several different elements in a piece of sketching. The primary task in the layout is to highlight the theme. It is necessary to try to balance the various elements in the picture and ensure the visual continuity between them. The purpose should be to naturally and quickly lead the bystander to the center of interest, while allowing the viewer to accept other details. The object of sketching in oil painting is the main component of a painting and the center of gravity of the painting. Other components need to be arranged around this center. There are many factors in the field of vision when sketching. Even if the composition is in the painters' field of vision, this does not mean that everything in their field of vision should be drawn. Careful selection must be made to find out which ones are useful for expressing the subject. If they juxtapose elements with similar effects in the picture, the elements will act independently, and the average is equal to dull, so it is necessary to choose the painting elements in the field of view according to the theme and center of interest.

It is not difficult to draw the sketch objects that the painters see, but to dare to deal with the picture boldly, it is difficult to refine and choose something for the needs of the picture. Therefore, cultivating the ability to process images is an important means to improve artistic accomplishment. With the correct guiding ideology, a way can be found to arrange the priority of the painting.

First, the painters should set the center of interest as the visual center of the picture, and the visual flow of other painting elements should match the layout of the subject. 
Second, when the drawn objects in the picture have similar sizes or shapes, they can use perspective to emphasize the theme.

Third, a good way to weaken the secondary elements in the foreground of the sketching object is to cut off part of its shape, or blur or weaken its characteristics.

\section{Induction and layout of forms and colors}

The artist Hans Hoffman said: "A painting, before it is anything, is the arrangement of shapes and colors in a two-dimensional space." This shows that before painting, the shapes and colors seen in the field of vision must be flattened, that is, all objects in the picture must be reduced to flat abstract shapes and color blocks. The painters need to summarize these shapes and color blocks from a macro perspective, and then design where should they be placed, how much share should they occupy, what brightness should appear, how should they be arranged to be unified and coordinated, or unified and confrontational, or how to paint more profoundly... These should be finalized on the small draft before the formal sketching.

1) Black-and-white layout: The black-and-white layout actually creates a simple beauty for the sketching. In the organization of black and white, it is a necessity to observe as a whole, and then use the keenest artistic perception to summarize the richness of colors. In specific sketching, the painters can summarize certain types of colors with similar brightness into black, white, and gray areas, and then incorporate colors that match the brightness according to the already summarized black, white and gray relationship. In the black and white layout, they must boldly choose and use black and white contrast to set off the subject. Everything serves the subject.

2) Color layout: The layout of colors is particularly important for oil painting sketching. Only when the relationship between the colors of the picture is designed can the sketching have vitality.

3) Induction of tones in the sketching composition: Tonal induction is an element of composition and an element of oil painting sketching. Henry Matisse said: "Color itself is not expressive. Only when it has been organized and its intensity protects the artist's feelings can it gain full expressiveness." Therefore, the painter needs to integrate the color subjectively and actively, so that the color can speak in its place. So how can they carry out the color layout? Firstly, through the overall observation and experience of the color atmosphere, a subjective color impression can be formed, which induces the emotion and poetry of color that are not for oneself, and forms a psychological color atmosphere.
For example, they are warm, lyrical, quaint, vigorous, abstract, intentional colors and so on. Then the painters need to design the tone of the entire color according to their feelings. Therefore, when designing the color block layout of lyrical tones, it is necessary to subjectively deal with or weaken the excessive contrast or very abrupt colors, so as to establish abstract color blocks that meet the needs of the aesthetic structure of the composition.

It is worth noting that the color management must first be established in the large black, white and gray layout that has been confirmed, so as to establish a powerful and stable picture. The painter must observe the color of the sketch object in advance to grasp the keynote. The colors need to be harmonious, and the painters need to compare to paint colors. When summarizing the tones, the painters need to try to achieve the unity of color changes, that is, the harmony and contrast of colors, just like every note must adapt to the melody of the entire movement. If the colors of a painting only focused on changing instead of unity, it will feel disorganized. And if only pay attention to the unity of colors and ignore the change of colors, it will also be monotonous and poor.

Colors are the most important carrier and language of oil painting sketching, and the success of color induction and processing affects the entire sketching process. The painters need to arrange the colors cleverly according to the composition to provide a guarantee for drawing a satisfactory sketching.

\section{E. Tempo and rhythm of composition in oil painting sketching}

The tempo and rhythm of composition are the main means of artistic treatment of sketching. Some people say that artistic processing only needs to be concentrated towards the end of sketching. This is a misunderstanding. As a matter of fact, the artistic process is like a shadow at the beginning of the composition.

In the sketching composition, many contradictory opposites on the screen produce rhythm. Tempo is an extremely important form of plastic art. It can create the beauty of the work. The various differences are organized in a single screen according to the author's conceived needs and adjusted and unified to produce tempo. This ability to regulate tempo is very important in composition. Tempo includes all aspects such as size, black and white, light and dark, strength, virtuality, weight, quantity, density, etc. All visual elements have problems with tempo and rhythm. If the tempo of the picture is mastered, the work will produce a kind of rhythm, and the visual beauty will be very attractive. Just as the painters can use strong lines to draw the observer's sight to interesting points, they can also maintain this visual movement in the picture, creating a 
sense of visual fluency and rhythm that can add musical vitality to the sketching. How to achieve this effect depends on the nature of the object being painted and the artist's artistic cultivation. The composition laws of virtuality and reality, strength and weakness, density, coordination and balance, confrontation and unity in the composition are all necessary means to make sketching closer to artistic effects.

\section{CONCLUSION}

In summary, composition plays a pivotal role in the steps of oil painting sketching, so it is necessary to attach great importance to it. Before sketching, the painters need to design the composition of the picture for the painted object, and to fully study and be familiar with the elements of the composition in order to complete an excellent sketching work. To have a good composition ability, the workers engaged in art creation are required to do a lot of field composition exercises and achieve a certain amount of accumulation, so as to be in good control of the composition. Therefore, it is of great significance to practice a lot of composition and draw inferences from one another, and the painters will definitely become more confident on the road of painting.

\section{References}

[1] Stefano Zuffi, Art and the Human Body [M]. Shandong Fine Arts Publishing House, 2003.

[2] Roberts I., The Art of Composition [M]. Shanghai People's Fine Arts Publishing House, 2012.

[3] Chang Ruilun, Painting Composition [M]. People's Fine Arts Publishing House, 2010. (in Chinese)

[4] Sun Tao, Beauty Save the World [M]. People's Fine Arts Publishing House, 2009. (in Chinese)

[5] Zheng Guangxu, The Structure of Points and Lines [M]. People's Fine Arts Publishing House, 2013. (in Chinese)

[6] Huang Keyou, A Brief Discussion on the Composition of Oil Painting [J]. Drama House, 2020(28):157-158. (in Chinese) 